\title{
Travelling Wave Fault Location Methods for Power Cables
}

\author{
Xia Zhao \\ School of Information Engineering, Nanjing Xiaozhuang University, Nanjing, P.R.China 210017 \\ email:244593412@qq.com
}

Keywords: power cable, fault location, travelling wave.

\begin{abstract}
: mathematic base of travelling wave is firstly analyzed in this paper and then one novel type of calculation method for travelling wave fault location for non-open circuit faults is proposed. The usage method in actual application is proposed to enhance the accuracy of traveling wave fault detection in an efficient manner.
\end{abstract}

\section{Introduction}

With the development of urbanization, power cables are widely used in the power distribution systems of large and medium cities and industrial and mining enterprises like oil fields and railways since they are safe, reliable, hidden, and durable and they are conducive to the urban beautification and layout of industrial and mining enterprises. The cable faults become more and more frequent in actual operation due to mechanical damage, insulation aging and material faults. It is rather difficult to locate the fault points since the cable laying is hidden. Non-open faults account for over ninety percent in many cable faults and it is the focus of research as to how to locate rapidly and accurately such faults. The theory base of travelling wave fault location and the shortcomings of traditional travelling wave fault location is analyzed from the mathematic perspective. Finally, one improved calculation method for such fault location is proposed for non-open circuit faults in combination with use method in actual application to improve the accuracy and reliability of travelling wave fault location.

\section{Equivalent Circuit of Cable Line}

Power cable is one type of transmission line. When the geometric length of transmission line is larger than or approximately equal to the wave length of the transmission signal, the transmission line is called long line. Since a large quantity of high frequency components are included in the travelling wave of pulse voltage (the narrower of the travelling wave of the pulse voltage, the steeper of the rise edge and the larger of the high frequency components) whose wave length is substantially small relative to the cable length, with the increase of voltage and frequency of the travelling wave transmitted, the displacement current and leakage current between core and sheath cannot be neglected. Besides, the high frequency component current produces high frequency alternating magnetic field which induces self-induced EMF, so as for the high frequency component in the pulse voltage, the transient travelling wave of cables can't be analyzed with simply with lumped parameters model, and the cables shall be taken as composition as shown in figure $1^{[1]}$ connected by numerous resistors, electric conductance, capacitors and inductance elements.

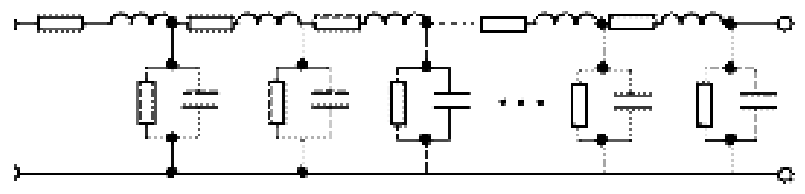

Fig.1. Transmission Line Distribution Parameter Model

\section{Mathematic analysis for travelling wave fault location}

One section is extracted as shown in figure 2 


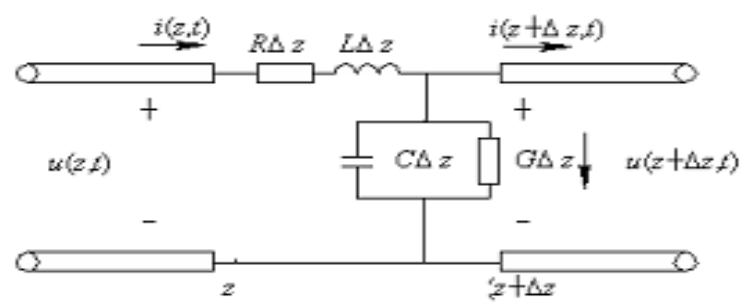

Fig.2.

From Kirchhoff's law, we can get:

$$
\left\{\begin{array} { l } 
{ u ( z , t ) - u ( z + \Delta z , t ) = R _ { 0 } \Delta z i ( z , t ) + L _ { 0 } \Delta z \frac { \partial i ( z , t ) } { \partial t } } \\
{ i ( z , t ) - i ( z + \Delta z , t ) = G _ { 0 } \Delta z u ( z + \Delta z , t ) + C _ { 0 } \Delta z \frac { \partial u ( z + \Delta z , t ) } { \partial t } }
\end{array} \Rightarrow \left\{\begin{array}{l}
-\frac{\partial u(z, t)}{\partial z}=R_{0} i(z, t)+L_{0} \frac{\partial i(z, t)}{\partial t} \\
-\frac{\partial i(z, t)}{\partial z}=G_{0} u(z, t)+C_{0} \frac{\partial u(z, t)}{\partial t}
\end{array}\right.\right.
$$

Based on theory of complex function, the sinusoidal can be represented as:

$$
\begin{aligned}
& \left\{\begin{array}{l}
u(z, t)=\operatorname{Re}\left[U(z) e^{j \omega t}\right] \\
i(z, t)=\operatorname{Re}\left[I(z) e^{j \omega t}\right]
\end{array}\right. \\
& U(z), I(z) \text { is copmplex function }
\end{aligned} \quad \Rightarrow\left\{\begin{array}{l}
-\frac{d U(z)}{d z}=\left(R_{0}+j \omega L_{0}\right) I(z)=Z I(z) \\
\frac{d I(z)}{d z}=\left(G_{0}+j \omega C_{0}\right) U(z)=Y U(z)
\end{array}\right.
$$

$Z$ is the unit length series impedance of transmission line, $Y$ is the unit length shunt admittance of transmission line, $Z$ is the length from the starting end of transmission line. The solution is as follows:

$$
\begin{aligned}
& \text { follows: } \\
& \begin{cases}U(z)=A_{1} e^{-\gamma z}+A_{2} e^{\gamma z} & Z_{0}=\sqrt{\frac{Z}{Y}}=\sqrt{\frac{R_{0}+j \omega L_{0}}{G_{0}+j \omega C_{0}}} \\
I(z)=\frac{1}{Z_{0}}\left(A_{1} e^{-\gamma z}-A_{2} e^{\gamma z}\right) & \gamma=\sqrt{Z Y}=\sqrt{\left(R_{0}+j \omega L_{0}\right)\left(G_{0}+j \omega C_{0}\right)} \\
& =\alpha+j \beta\end{cases}
\end{aligned}
$$

Substitute $U(0)=U_{L}, I(0)=I_{L} \quad$ into formula $\left(^{*}\right)$ and we get: $\left\{\begin{array}{l}A_{1}=\left(U_{L}+Z_{0} I_{L}\right) / 2 \\ A_{2}=\left(U_{L}-Z_{0} I_{L}\right) / 2\end{array}\right.$

The instant representation of voltage and current on the transmission line is (the coordinate direction is the source pointing to the load)

$$
u(z, t)=\operatorname{Re}\left[U(z) e^{j \omega t}\right]=A_{1} e^{-\alpha z} \cos (\omega t-\beta z)+A_{2} e^{\alpha z} \cos (\omega t+\beta z)=u_{+}+u_{-}
$$

Among which:

$$
\left.\alpha=\sqrt{1 / 2\left[\sqrt{\left(R_{0}^{2}+\omega^{2} L_{0}^{2}\right)\left(G_{0}^{2}+\omega^{2} C_{0}^{2}\right)}+\left(R_{0} G_{0}-\omega^{2} L_{0} C_{0}\right)\right.}\right] \quad \beta=\sqrt{1 / 2\left[\sqrt{\left(R_{0}^{2}+\omega^{2} L_{0}^{2}\right)\left(G_{0}^{2}+\omega^{2} C_{0}^{2}\right)}-\left(R_{0} G_{0}-\omega^{2} L_{0} C_{0}\right)\right]}
$$

In the transmission direction of wave, the distance of two points with $2 \pi$ of phase difference is wave length of $\lambda$, then we can get $\omega t-\beta(z+\lambda)=\omega t-\beta z-2 \pi, \lambda=2 \pi / \beta$, travelling wave speed $v=\lambda f=\omega / \beta$. As shown in above mathematic analysis, during static state, the voltage at any point of transmission line is formed by superposition of incident wave voltage $u_{+}$and reflected wave voltage $u_{-}$. The incident wave is sinusoidal travelling wave passing the amplitude from the starting point to the ending point with exponential decay; the reflected wave is the sinusoidal travelling wave passing the amplitude from the ending point to the starting point with exponential decay. When pulse voltage is just loaded to the starting point of cable, the cable is charging and then moves with the charge transmission to the ending point of cable. In this way, incident wave voltage of $u_{+}$and current of $i_{+}$are generated on each point of the cable. Since the charge does not reach the ending point, $u_{-}$and $i_{-}$are not generated for the moment. When the charge reaches the ending point, since boundary conditions of ending point (or the fault point) have to be met, if boundary condition at ending point during short circuit is that the voltage is 0 , and the boundary condition at ending point during open circuit is that the current is 0 , the reflection to the charge will definitely be produced, which generates reflected voltage wave and current wave. This transient 
process is where the theory basis is for our travelling wave fault location.

\section{Traditional travelling wave fault location method}

Since the reliability and accuracy of travelling wave fault location are not affected in theory by type of line, resistance of fault and system on both sides, this method remains the hot point of study. Currently, travelling wave fault location is widely applied on site, including low voltage pulse reflection, pulse voltage and pulse current, etc. ${ }^{[2]}$

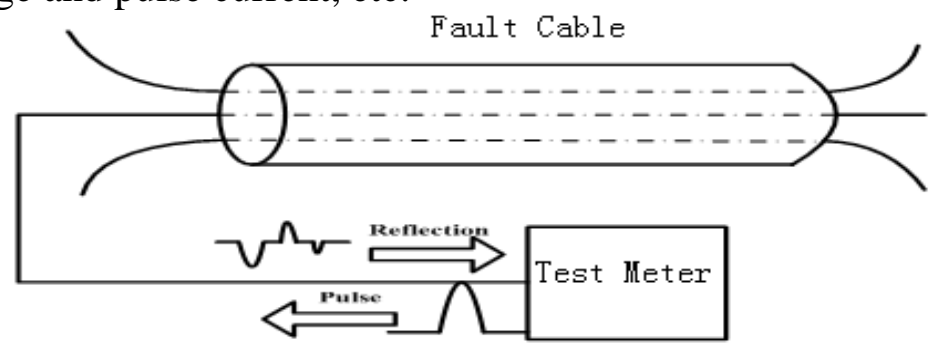

Fig.3. Schematic of fault location system with pulse reflection

The figure 3 shows the schematic of fault location system with pulse reflection. One pulse wave is generated in the test meter to be loaded on the cable being tested. When the pulse wave meets the mismatch point of cable characteristic impedance, one reflected wave signal will be generated (based on the transmission line principle) and the test meter will acquire these two signals (transmitted wave and reflected wave) at the test end of the cable to calculate the distance between the fault point and test end. Set the time difference between the transmitted wave and reflected wave to be $\Delta t$, the wave speed of travelling wave in the cable is $v$, and the calculation formula for the fault point distance is

$L=\Delta t \times v / 2$

The accuracy of travelling wave fault location depends on the transmission speed of the wave in the cable being tested and the accuracy of time difference of arrival at the test end between the incident wave and the reflected wave. The wave speed $v$ of cable is only related to the feature of insulation medium and has nothing to do with the material and section area of the core of conductor. Table 1 shows the reference values of pulse wave speeds in cables with different insulation media.

Table 1 Reference values of pulse wave speeds in cables

\begin{tabular}{c|c|c|c|c}
\hline $\begin{array}{c}\text { Insulation medium } \\
\text { of cable }\end{array}$ & $\begin{array}{c}\text { XLPE } \\
3 \text { cores }\end{array}$ & $\begin{array}{c}\text { XLPE } \\
\text { Single core }\end{array}$ & PVC & Oiled paper \\
\hline$v(\mathrm{~m} / \mu \mathrm{s})$ & 220 & 190 & $170-200$ & $160-170$ \\
\hline
\end{tabular}

As for the time difference $\Delta t$ between incident wave and reflected wave, the wave shape signal acquired by the high speed acquisition card of test meter will be displayed by oscilloscope and then read. As shown by formula (1), the present travelling wave fault location is related to the wave speed and the wave speed adopts in most cases the empirical wave speed in table 1 . Actually, the transmission of travelling wave inside the cable includes rich frequency components and traveling waves with different frequencies have different speeds ${ }^{[2]}$ : the higher the frequency and the faster the transmission speed. Therefore, one empirical value is used to replace all the speeds of travelling waves, which will definitely cause large error of fault location.

\section{One improved travelling wave fault location solution}

As shown by the above analysis, the wave speed of travelling wave inside the cable determines directly the accuracy of the fault location. If the influence of unstable wave speed can be eliminated, the error of the fault location will be definitely reduced. In non-open circuit fault, the travelling wave has reflection and transmission which are shown in fig. 4. 


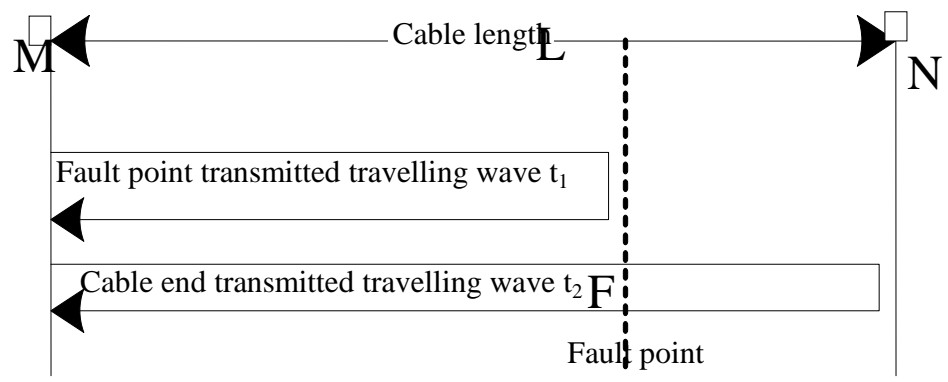

Fig.4. Traveling wave transmission process

The total length of cable is $\mathrm{L}$, point $\mathrm{M}$ is the end where the pulse signal is loaded, $\mathrm{N}$ is the ending point of the cable. Assume the fault generation point of the cable meets $|F-M|>|F-N|$, i.e. the fault point is much more distant from point $\mathrm{M}$, the arrival time of the first two wave heads are $t_{1}$ and $t_{2}$ respectively. Base do the reflection principle, the reflected wave at the fault point will arrive at the point $\mathrm{M}$ before the reflected wave at the opposite end of the line. Therefore, $t_{1}$ is the time for the reflected wave at the fault point to arrive at the test end and the $t_{2}$ is the time for the reflected wave at the opposite end of the line to arrive at the test end, so: $\left\{\begin{array}{l}l_{1}=\frac{t_{1} v}{2} \\ 2 L=v t_{2}\end{array}\right.$, We get $l_{1}=\frac{t_{1}}{t_{2}} L$ (2) Similarly, when $|F-M|<|F-N|$, i.e. when the fault point is much more distant from point $\mathrm{N}$, it is only necessary to add the pulse voltage signal and record again $t_{1}$ and $t_{2}$. In real application, (1) is used firstly to preliminarily estimate the distance $l_{10}$ between the fault point and measurement end, and then select whether the measurement end is $\mathrm{M}$ or $\mathrm{N}$ based on the size of $l_{10}$ and $L / 2$. After measurement end is selected, record $t_{1}$ and $t_{2}$, and formula (2) is used to calculate once again the fault point distance.

\section{Conclusion}

Aimed at the non-open circuit fault, the traveling wave fault location method proposed here can locate rapidly and accurately the fault point. This method eliminates the influence of speed of travelling wave, improves the accuracy of fault location. However, actual length is required by this method and when the cable length is not accurately known, error will also occur in this method. Therefore, traditional method shall also be used as supplementation in real application to realize accurate location of cable fault points.

\section{References}

[1] Rihong Jiang. XLPE power cables (version 2) [M]. China Electric Power Press, 2009, 89-109

[2] Bingyin Xu, Shengxiang Li, Zongjun Chen. Detection technology on power cable fault [M]. China Machine Press, 2001, 32-53

[3] Jian Tan, Xiangxun Chen, Jianchao Zheng. Study on dispersion of travelling wave in transmission line [J]. Proceedings of the CSEE, 1999, 19(9):27-30

[4] Zongshu Wei. Probability theory and mathematical statistics course [M]. Higher Education Press, 2008

[5] Peng Zou, Xiaoping zhou. Electromagnetic fields and waves [M]. Third edition. Beijing: Higher Education Press, 2008 